\title{
Principais cuidados de enfermagem aos pacientes portadores de Lúpus Eritematoso Sistêmico: relato de experiência
}

\author{
Main nursing care for systemic lupus erythematosus patients: experience report
}

Atención de enfermería principal para pacientes con lúpuseritematoso sistémico: informe de experiencia

\section{Sarah Cristianny Dantas dos Santos ${ }^{1^{*}}$, Priscila Cristina da Silva Thiengo ${ }^{2}$, Cristiane Helena Gallasch $^{3}$, Ariane da Silva Pires ${ }^{4}$, Helena Ferraz Gomes ${ }^{5}$, Eugenio Fuentes Pérez Júnior ${ }^{6}$}

Como citar esse artigo. dos Santos, SCD; Thiengo, PCS; Gallasch, $\mathrm{CH}$; Pires, AS; Gomes, HF; Junior, EFP. Principais cuidados de enfermagem aos pacientes portadores de Lúpus Eritematoso Sistêmico: relato de experiência. Revista Pró-UniverSUS. 2019 Jul/Dez.; 10 (2): 39-47.

\section{Resumo}

O Lúpus Eritematoso Sisteêmico (LES) é uma doença inflamatória crônica, multissistêmica, de natureza auto-imune, caracterizada pela presença de diversos auto-anticorpos.Trata-se de um relato de experiência vivido por uma residente de enfermagem entre os meses deabril de 2017 a abril de 2018 em uma enfermaria de clínica médica, sustentadoa partir do levantamento de dados na literatura. Os principais cuidados de enfermagem ofertados foram: promover ambiente calmo para sono e repouso; controlar rigorosamente o balanço hídrico; verificar peso diariamente em jejum; avaliar estado geral e nutricional; manter níveis pressóricos adequados; avaliar acessos venosos e outros dispositivos invasivos quanto a sinais flogísticos; avaliar dor, promover ambiente tranquilo e administrar analgésico; evitar leitos com incidência direta de luz solar; avaliar resultados de exames laboratoriais; oferecer cremes a base de uréia para hidratação da pele; incentivar e orientar sobre o autocuidado; fornecer informações suficientes sobre a doença e o tratamento e oferecer apoio emocional para enfrentamento das mudanças corporais. Encontraram-se apenas dois artigos na literatura que citavam cuidados de enfermagem aos portadores de LES. Os cuidados de enfermagem realizados corroboram com os dois artigos encontrados, porém nenhum deles citou sobre o cuidado de evitar leitos com incidência direta de luz solar. A experiência enquanto residente de enfermagem proporcionou domínio de competências técnicas e científicas para atuação na enfermagemclínica.

Palavras-chave: Lúpus Eritematoso Sistêmico, Cuidados de enfermagem, Diagnóstico de Enfermagem, Enfermagem

Afiliação dos autores:1. Enfermeira e residente do programa de clínica médica/Faculdade de Enfermagem da Universidade do Estado do Rio de Janeiro, RJ, Brasil. Email: sarah. santosenf@gmail.com ORCID: https://orcid.org/0000-0003-4179-6693

2. Enfermeira, mestre em Enfermagem e professora assistente/Faculdade de Enfermagem da Universidade do Estado do Rio de Janeiro, RJ, Brasil. Email: jacqueline.enfa2019@ gmail.com ORCID: https://orcid.org/0000-0003-0840-4838

3. Enfermeira,doutora em Enfermagem e professora adjunta/Faculdade de Enfermagem da Universidade do Estado do Rio de Janeiro, RJ, Brasil. Email: palomamiglu@gmail.com ORCID:Orcid: https://orcid.org/0000-0002-0823-0818

4. Enfermeira,doutora em Enfermagem e professora adjunta/Faculdade de Enfermagem da Universidade do Estado do Rio de Janeiro, RJ, Brasil. Email: renespezani@gmail.com ORCID:Orcid: https://orcid.org/0000-0003-1123-493X

5. Enfermeira e residente do programa de clínica médica/Faculdade de Enfermagem da Universidade do Estado do Rio de Janeiro, RJ, Brasil. Email: renespezani@gmail.com ORCID:Orcid: https://orcid.org/0000-0001-6089-6361

6. Enfermeiro, doutor em enfermagem e professora assistente/Faculdade de Enfermagem daUniversidade do Estado do Rio de Janeiro, RJ, Brasil. Email: renespezani@gmail.com ORCID:Orcid:https://orcid.org/0000-0003-4611-0443 


\section{Resumen}

El objetivo de este estudio es informar los principales cuidados de enfermería a pacientes con lupus eritematoso sistémico (LES) realizados durante la residencia de enfermería. El LES es una enfermedad inflamatoria multisistémica crónica de naturaleza autoinmune, caracterizada por la presencia de varios autoanticuerpos. Este es un informe de experiencia vivido por un residente de enfermería desde abril de 2017 hasta abril de 2018 en una sala de clínica médica, respaldado por la recopilación de datos en la literatura. Los principales cuidados de enfermería ofrecidos fueron: promover un ambiente tranquilo para dormir y descansar; controlar estrictamente el balance de agua; revise el peso en ayunas diariamente; evaluar el estado general y nutricional; mantener niveles adecuados de presión arterial; evaluar el acceso venoso y otros dispositivos invasivos para detectar signos flogísticos; evaluar el dolor, promover un ambiente pacífico y administrar analgésicos; evitar camas con incidencia directa de luz solar; evaluar resultados de pruebas de laboratorio; ofrecer cremas a base de urea para hidratar la piel; alentar y guiar el autocuidado; Brindar información suficiente sobre la enfermedad y el tratamiento y brindar apoyo emocional para hacer frente a los cambios corporales. Solo había dos artículos en la literatura que citan la atención de enfermería para pacientes con LES. El cuidado de enfermería realizado corrobora los dos artículos encontrados, pero ninguno menciona el cuidado de evitar camas con incidencia directa de luz solar. La experiencia como residente de enfermería proporcionó el dominio de las habilidades técnicas y científicas para la práctica clínica de enfermería.

Palabras clave: Lupus Eritematoso Sistémico, Atención de Enfermería, Diagnóstico de Enfermería, Enfermería.

\section{Introdução}

Este artigo trata sobre os cuidados de enfermagem ao paciente portador de Lúpus Eritematoso Sistêmico (LES), uma patologia relevante para o contexto de saúde no Brasil e no mundo, tendo em vista sua natureza autoimune, crônica e com potencial para gerar complicações ao paciente. O objetivo deste trabalho é elencar os cuidados de enfermagem ofertados aos pacientes portadores de LES através de um relato de experiência e também identificar na literatura esses cuidados para que haja uma comparação entre a teoria e a prática.

O Lúpus Eritematoso Sistêmico é uma doença inflamatória crônica, multissistêmica, de natureza auto-imune, caracterizada pela presença de diversos auto-anticorpos. Sua etiologia não é esclarecida e seu desenvolvimento está ligado a alterações imunológicas, predisposição genética e fatores ambientais como luz ultravioleta, vírus e alguns medicamentos. ${ }^{1,2}$

Trata-se de uma doença potencialmente grave devido à sua característica inflamatória, que provoca vasculite de médios e de pequenos vasos e todos os órgãos estão sujeitos a danos, inclusive aqueles cuja função é vital para a sobrevivência, como coração, pulmão, rins e sistema nervoso central. No entanto, com as novas possibilidades terapêuticas, as crises agudas da doença puderam ser controladas, reduzindo assim a mortalidade oriunda da vasculite e assumindo um caráter crônico. ${ }^{2}$

O diagnóstico de LES é feito quando o paciente apresenta quatro ou mais dos onze critérios estabelecidos pelo Colégio Americano de Reumatologia em 1982: eritema malar, eritema discoide, fotossensibilidade, úlcera oral, artrite, serosite, distúrbios renais, neurológicos, hematológicos e ocorrência de anticorpos antinucleares. ${ }^{3}$

Pacientes portadores de LES possuem um acréscimo no risco de adquirir infecções: redução de linfócitos T CD4+, deficiência de componentes do sistema complemento, neutropenia elinfopenia. Dessa maneira a fase ativa da doença é fator de risco para infecções, principalmente quando envolve órgãos como rim e de sistema nervoso central. O risco se deve pelo maior uso de imunossupressores, que destroem as defesas normais do corpo humano e favorecem a agressão por microrganismos. ${ }^{4}$

O LES pode afetar pessoas de qualquer sexo, raça e idade, no entanto, as mulheres são muito mais atingidas e a ocorrência em indivíduos mestiços e afrodescendentes é mais frequente. No Brasil não há dados significativos consolidados acerca do lúpus eritematoso sistêmico, mas acredita-se que uma entre 1.700 mulheres é acometida pela doença e estimativas apontam que existem cerca de 65.000 pessoas com convivendo com Lúpus, sendo a maioria mulheres. ${ }^{5}$

Em diferentes locais do mundo, a incidência estimada é de 1 a 22 novos casos a cada 100.000 pessoas por ano e a prevalência varia entre 7 a 160 casos a cada 100.000 pessoas por ano. No Brasil, estima-se que a incidência de LES é de 8,7 novos casos a cada 100.000 pessoas. $^{2}$

Ainda no contexto epidemiológico, mulheres entre 15 e 45 anos de idade possuem maior incidência de lúpus eritematoso sistêmico. Nesta faixa etária, a razão de incidência nas mulheres está em cerca de 6:1, ao passo que a razão de incidência entre homens é de 9:1. Além disso, pessoas de raça negra têm quatro vezes mais chances de desenvolver a doença quando comparadas com pessoas de raça branca. ${ }^{6}$

Durante o processo de hospitalização, o cliente acometido pelo Lúpus necessita de uma equipe multiprofissional, podendo ser constituída, em sua maioria, por: nutricionistas, médicos, enfermeiros e técnicos de enfermagem. À equipe de enfermagem cabe a responsabilidade de, por meio da sistematização da assistência, planejar, implementar e avaliar os cuidados em consonância com as necessidades do cliente, detectando precocemente sinais e sintomas de complicações, além de contribuir para seu bem estar. ${ }^{7}$

A Sistematização da Assistência de Enfermagem 
(SAE) é a dinâmica das ações sistematizadas e interrelacionadas, que viabiliza a organização da assistência de enfermagem. Representa uma abordagem de enfermagem ética e humanizada, dirigida à resolução de problemas, atendendo às necessidades de cuidados de saúde e de enfermagem de uma pessoa. O Processo de Enfermagem é um método sistematizado de prestação de cuidados que visa a obtenção de resultados desejados. É sistemático por se constituir de etapas, durante as quais, são dados passos deliberados para potencializar a eficiência e atingir resultados benéficos. ${ }^{7}$

Partindo desses princípios, o LES e suas complicações apresentam umquadro interessante para intervenções de enfermagemvoltadas à redução dos desfechos clínicos de descompensação e àmelhora da qualidade de vida dos pacientes afetados. Portanto, a residência de enfermagem na área de clínica médica possibilitou a experiência da assistência à saúde a pacientes acometidos por inúmeras patologias e, inevitavelmente, a pacientes portadores de LES que necessitavam de internação hospitalar. Portanto surgiram questionamentos sobre quais eram os principais cuidados de enfermagem que deveriam ser ofertados a esses clientes afim de minimizar complicações da doença e identificar problemas reais e potenciais de cada um. Baseando-se na prática assistencial diáriapercebeuse que quanto maior o conhecimento sobre o LES e os cuidados voltados para essa doença, maior era a qualidade da assistência de enfermagem ofertada.

Este estudo tende a ampliar a discussão e reflexão sobre o cuidado de enfermagem ao paciente portador de lúpus, tendo em vista a pouca produção encontrada durante a revisão de literatura. Especificamente para o ensino, o estudo pretende possibilitar novas estratégias para que o cuidado de enfermagem tenha critérios cientificamente comprovados afim de que o paciente tenha menor risco relacionado à assistência, além de contribuir para o levantamento de informações importantes e fomentando o pensamento crítico.

\section{Materiais e método}

Trata-se de uma pesquisa de naturezadescritiva, exploratória, com abordagemqualitativa, do tipo relato de experiência, realizada em um hospital universitário do Rio de Janeiro. O relato foi baseado na experiência de uma residente de enfermagem do programa de clínica médica em relação aos cuidados de enfermagem aos pacientes portadores de LES. O objetivo deste estudo écitar os cuidados de enfermagem realizados aos pacientes portadores de LES internados em um hospital público.

A experiência ocorreu no período de Abril de 2017 a Abril de 2018 em uma enfermaria de clínica médica do Hospital Universitário Pedro Ernesto (HUPE) localizado na Zona Norte do município do Rio de Janeiro. A pósgraduação latu sensu é promovida através do programa deresidência em enfermagem. A modalidade de residência é oferecida pela Faculdade de Enfermagem da Universidade do Estado do Rio de Janeiro, onde o HUPE é o principal campo de treinamento em serviço para os residentes que ingressam anualmente através de um processo seletivo.

Ao desenvolver este relato de experiência, houve a necessidade de embasamento teórico da literatura e optou-se, portanto, pelo levantamento do estado da arte em bases de dados nacionais e internacionais com o objetivo de elencar os cuidados de enfermagem aos pacientes portadores de LES para melhor compreensão acerca do tema e para que houvesse uma comparação entre os cuidados descritos na literatura e os cuidados realizados na prática da assistência à saúde.

O levantamento de artigos iniciou-se em Fevereiro de 2019 nas seguintes bases de dados: Biblioteca Virtual em Saúde (BVS) direcionando as buscas nas bases de dados: Medical Literature Analysis and Retrieval System Online (MEDLINE), Literatura Latino-americana e do Caribe em Ciências da Saúde (LILACS), Base de Dados em Enfermagem (BDENF) e Pubmed, tendo como critérios de inclusão: artigos completos e disponíveis gratuitamente nas bases de dados utilizadas, disponíveis nos idiomas português, inglês ou espanhol. Foram excluídos editoriais, cartas ao editor, resumos, opinião de especialistas e revisões. As informações extraídas dos artigos selecionados foram: título do artigo, base de dados indexada, autor, país de origem, idioma, ano de publicação, local de realização da pesquisa, aspectos metodológicos, resposta a questão norteadora e principais conclusões.

Os descritores indexados no DeCS utilizados no portal da BVS foram: Lúpus Eritematoso Sistêmico, Cuidados de Enfermagem, Diagnóstico de Enfermagem e Enfermagem. Para a pesquisa na base de dados Pubmed foram utilizados os descritores equivalentes indexados no MeSH: Lupus Erythematosus Systemic, Nursing Care, Nursing diagnosis e Nursing.

Referenciando a Resolução n ${ }^{\circ} 466 / 12$ do Conselho Nacional de Saúde sobre a ética em pesquisa com seres humanos, faz-se necessário esclarecer que não foram utilizados dados de pacientes ou de seus prontuários no estudo. A experiência foi desenhada a partir da observação direta da pesquisadora em seu ambiente de trabalhosem nenhuma anotação ou registro. Sendo assim, por se tratar de um relato de experiência, não houve aplicação de Termo de Consentimento Livre e Esclarecido. ${ }^{8}$

\section{Resultados e discussão}

A experiência enquanto residente de enfermagem 
proporcionou maior conhecimento sobre os desafios da atuação do enfermeiro frente a patologias potencialmente graves, como o Lúpus Eritematoso Sistêmico, que requer maior aprofundamento sobre o tema para que possa ser melhor abordado. Portanto, o tópico de resultados foi subdividido entre o estado da arte, que forneceu suporte teórico, e a descrição do relato de experiência.

\section{Estado da Arte}

Foram executados os seguintes cruzamentos para pesquisa na Biblioteca Virtual em Saúde: Lúpus Eritematoso Sistêmico AND Cuidados de Enfermagem, Lúpus Eritematoso Sistêmico AND Diagnóstico de Enfermagem e Lúpus Eritematoso Sistêmico AND Enfermagem. Foram executados cruzamentos equivalentes para a pesquisa na base de dados Pubmed: Lupus Erythematosus Systemic AND Nursing Care, Lupus Erythematosus Systemic AND Nursing Diagnosis, Lupus Erythematosus Systemic AND Nursing. A síntese dos cruzamentos de dados pode ser visualizada na figura 1.

Diante da pesquisa realizada nas bases de dados, o resultado obtido na literatura sobre o conhecimento pertinente aos cuidados de enfermagem aos portadores de LES foi limitado. Em quatro bases de dados distintas foram selecionados apenas dois artigos que responderam à questão norteadora. Foram obtidos dois artigos de fontes primárias publicados nos anos de 2011 e 2013. Em relação aos periódicos nos quais foram publicados, um é estrangeiro e outro nacional: American Academy of Nurses Practitioners e Revista Brasileira de Ciências da Saúde, respectivamente.

A partir do resultado obtido, o artigo 1 revelou 17 cuidados de enfermagem, ao passo que o artigo 2 revelou 7 cuidados de enfermagem. Ambos artigos se referem a cuidados de enfermagem independente da metodologia utilizada, amostra estudada e análise de dados. No Quadro 1observam-se os cuidados de enfermagem aos pacientes portadores de LES extraídos dos artigos selecionados.

$\mathrm{O}$ artigo 1 trata de um estudo de caso realizado através de um instrumento de coleta de dados utilizado por enfermeiros durante a admissão do paciente. Após anamnese e exame físico do cliente, foram elencados treze diagnósticos de enfermagem e posteriormente realizados os cuidados de enfermagem mencionados acima. Percebe-se que os cuidados de enfermagem descritos perpassam os níveis de promoção e proteção à saúde. ${ }^{9} \mathrm{O}$ artigo 2 diz respeito a uma revisão bibliográfica que tem como objetivo revisar aspectos genéticos, sintomas e opções de tratamento para os pacientes portadores de LES onde os cuidados de enfermagem elencados também tratam de educação e promoção à saúde. ${ }^{10}$

Ambos os artigos citaram cuidados de enfermagem

\author{
Lupus Eritematoso Sistêmico AND \\ Cuidados de Enfermagem / Lupus \\ Eritematosus Systemic AND Nursing \\ Care \\ BVS: 14 artigos filtrados $->2$ artigos selecionados
}

Pubmed: 1444 artigos filtrados -> 0 artigos selecionados

BVS: 4 artigos filtrados -> 0 artigos selecionados

Lúpus Eritematoso Sistêmico AND Diagnóstico de Enfermagem / Lupus Eritematosus Systemic AND Nursing Diagnosis

Pubmed: 996 artigos filtrados -> 0 artigos selecionados

Lupus Eritematoso Sistêmico AND Enfermagem / Lupus Eritematosus Systemic AND Nursing
BVS: 23 artigos filtrados $->0$ artigos selecionados

Pubmed: 514 artigos filtrados -> 0 artigos selecionados

Figura 1. Cruzamentos nas bases de dados. Fonte: própria autora, 2019. 
Quadro 1. Cuidados de enfermagem aos portadores de LES descritos na literatura. Rio de Janeiro, 2019.

\begin{tabular}{|c|c|}
\hline $\begin{array}{l}\text { Artigo l } \\
\text { Revista Brasileira de ciências da Saúde } \\
\text { (2013). }\end{array}$ & $\begin{array}{l}\text { Artigo } 2 \\
\text { American Academy of Nurses Practitioners } \\
\text { (2011). }\end{array}$ \\
\hline Esclarecer os beneficios da atividade física & Encorajar proteção solar \\
\hline $\begin{array}{l}\text { Encorajar a realização de exercícios de } \\
\text { condicionamento }\end{array}$ & $\begin{array}{l}\text { Esclarecer sobre drogas que induzem sintomas } \\
\text { semelhantes aos do lúpus }\end{array}$ \\
\hline $\begin{array}{l}\text { Estimular a ingesta de } 2 \text { litros de água por dia e } \\
\text { alimentos ricos em fibra }\end{array}$ & Realizar o controle da pressão arterial \\
\hline $\begin{array}{l}\text { Investigar todas as vias invasivas a cada } 24 \mathrm{~h} \\
\text { quanto aos sinais flogísticos }\end{array}$ & Estimular o controle da nutrição \\
\hline Avaliar os achados laboratoriais & Estimular a parada do tabagismo \\
\hline $\begin{array}{l}\text { Ensinar a reconhecer os sinais e sintomas da } \\
\text { infecção }\end{array}$ & Incentivar a prática de exercício \\
\hline $\begin{array}{l}\text { Avaliar hábitos de higiene corporal e instruir a } \\
\text { prática }\end{array}$ & Conferir as imunizações \\
\hline Manter níveis pressóricos adequados & \\
\hline Orientar o uso de hipotensores & \\
\hline $\begin{array}{l}\text { Pesar diariamente registrando a perda ou ganho } \\
\text { de peso }\end{array}$ & \\
\hline $\begin{array}{l}\text { Conversar em termos compreensiveis sobre } \\
\text { LES e o tratamento }\end{array}$ & \\
\hline Avaliar a integridade cutânea a cada $4-8$ horas & \\
\hline Explicar sobre a importância da higiene oral & \\
\hline $\begin{array}{l}\text { Estabelecer vínculos de confiança através de } \\
\text { conversas amigáveis }\end{array}$ & \\
\hline Encorajar a verbalizar reações e sentimentos & \\
\hline $\begin{array}{l}\text { Avaliar alterações na aparência e seu } \\
\text { significado para o paciente }\end{array}$ & \\
\hline $\begin{array}{l}\text { Estimular a participação da paciente em seu } \\
\text { tratamento }\end{array}$ & \\
\hline
\end{tabular}


relacionados ao controle da pressão arterial e à pratica de atividade física, o que leva a crer que são cuidados de enfermagem prioritários aos portadores de LES, uma vez que esses pacientes comumente apresentam hipertensão arterial sistêmica, sobrepeso e fadiga, sintomas que podem ser reduzidos e controlados através da prática deeducação em saúde.

\section{Descrição da Experiência}

O cenário prático da residência de enfermagem da Universidade do Estado do Rio de Janeiro (UERJ) ocorreu no Hospital Universitário Pedro Ernesto (HUPE), localizado na região Norte do município do Rio de Janeiro. Trata-se de um hospital público que em 1975 deixou de acompanhar apenas o estudo de raridades clínicas e passou a funcionar como hospital de atendimento geral com mais de 60 especialidades e subespecialidades onde funcionam cerca de 525 leitos, adequando-se às necessidades da população do Rio de Janeiro. A missão do HUPE é prestar assistência integrada, humanizada e de excelência à saúde, sendo agente transformador da sociedade através do ensino, pesquisa e extensão. Sua visão é ser um referencial de excelência no ensino, pesquisa e assistência na área da saúde do Estado do Rio de Janeiro e tem como valores o respeito à vida e à dignidade do ser humano, ética nas relações, transparência na divulgação das ações, competência técnica, trabalho em equipe, pioneirismo e responsabilidade Social. ${ }^{11}$

O HUPE possui 4 enfermarias de clínica médica nomeadas 11 e 12,13 e 14, 15 e 16 e 17 e 18 cujas características principais são a divisão de leitos em alas masculina e feminina em cada enfermaria, bem como esquipes médicas e de enfermagem responsáveis por cada uma delas. Os perfis mais comuns de pacientes internados eram: portadores de doenças crônicas não transmissíveis tais como hipertensos, diabéticos, nefropatas, pacientes acometidos por doenças reumatológicas e hematológicas. A enfermaria do cenário prático do primeiro ano de residência foi a 15 e 16 e entre as quatro enfermarias de clínica médica era a única a ter dois dos seus doze leitos reservados para estabilização de pacientes com quadro clínico agravado atendidos pelo plantão geral, unindo em uma enfermaria pacientes de clínica médica e de urgência e emergência.

Um importante adendo ao relato de experiência diz respeito ao momento de crise político-econômica vivenciada intensamente no Estado do Rio de Janeiro no ano de 2017, onde a saúde pública foi sumariamente afetada pela falta de repasse de verbas ao HUPE, o que implicou diretamente na redução do número de leitos do hospital, escassez de recursos materiais e humanos, assim como uma onda de grande insatisfação por parte de todas as classes profissionais que atuavam para o funcionamento da instituição.

Diante de todo o exposto, a experiência vivenciada com os portadores de LES era iniciada a partir da admissão do paciente na enfermaria 15 e 16, cujos leitos de internação eram divididos em 5 na ala masculina e 5 na ala feminina.A equipe multiprofissional era composta por médicos, enfermeiros, técnicos de enfermagem, nutricionistas e, eventualmente, assistentes sociais, fisioterapeutas e psicólogos. A equipe de enfermagem diária era formada por 1 enfermeiro, 3 técnicos de enfermagem e 6 enfermeiras residentes.

A internação do paciente com diagnóstico de LES era realizada baseada na necessidade de tratamento diante do quadro clínico que ele apresentava e principalmente nas complicações ocasionadas pela patologia que por vezes acometia diversos órgãos. Observou-se que dentre as principais causas de internação hospitalar dos portadores de LES destacavam-senefrite lúpica, artrite, serosite e distúrbios hematológicos. Em relação a características dos pacientes, observou-se prevalência de LES apenas no sexo feminino, raça branca e idade entre 20 e 40 anos.Os principais sinais e sintomas que acometiam os pacientes eram fadiga, disúria, dor, hipertensão, edemas de membros inferiores, artralgia, febre, rash cutâneo e perda ponderal.

As condutas de enfermagem foram amparadas pela Sistematização da Assistência de Enfermagem (SAE) que é dividida em 5 etapas inter-relacionadas executadas pelo enfermeiro: 1) Histórico de Enfermagem, 2) Diagnóstico de enfermagem, 3) Planejamento, 4) Implementação e 5) Avaliação.

A avaliação inicial do pacienteadmitido no setor era realizada a partir do histórico de enfermagem com instrumento de coleta de dados baseado na taxonomia NANDA-I, constituído por dados pessoais e sociais, história patológica pregressa, histórico de doença familiar, história da doença atual, histórico farmacológico, as principais queixas, internações anteriores, cirurgias prévias, alergias, estado nutricional, estado emocional, exame físico, entre outros. Em seguida eram elencados os principais diagnósticos de enfermagem, levantando problemas reais e potenciais do cliente. No Quadro 2 estão destacados os diagnósticos de enfermagem mais utilizados para os portadores de LES da enfermaria 15 e 16.

A partir da análise dos dados coletados através do Histórico de Enfermagem, os diagnósticos de enfermagem eram traçadosno sentido de elaborar objetivos e metas que eram transformadas em prescrição de enfermagem como um planejamento do cuidado para serem alcançados na assistência ao paciente portador de LES.Diante dos diagnósticos de enfermagem elencados, os principais cuidados de enfermagem praticados na enfermaria 15 e 16 para os pacientes portadores de LES estão representados no Quadro 3. 
Quadro 2. Principais diagnósticos de enfermagem. Rio de Janeiro, 2017-2018.

\begin{tabular}{|l|}
\hline \multicolumn{1}{|c|}{ Diagnósticos de Enfermagem relacionados aos pacientes portadores de LES } \\
\hline -Fadiga relacionada à falta de condicionamento fisico e privação do sono \\
\hline -Dor aguda relacionada a agentes causadores de lesão \\
\hline $\begin{array}{l}\text {-Integridade da pele prejudicada relacionada à alteração no metabolismo, circulação } \\
\text { prejudicada e imunodeficiência }\end{array}$ \\
\hline -Intolerância à atividade relacionada a estilo de vida sedentário e fraqueza generalizada \\
\hline -Risco de desequilíbrio eletrolítico relacionado à disfunção renal e mecanismo regulador \\
comprometido \\
\hline -Distúrbio na imagem corporal relacionado à doença e regime de tratamento \\
\hline $\begin{array}{l}\text {-Falta de adesão relacionada a conhecimento insuficiente sobre o regime, motivação } \\
\text { insuficiente e duração prolongada do regime }\end{array}$
\end{tabular}

Quadro 3. Principais cuidados de enfermagem - Rio de Janeiro - 2017 e 2018.

\begin{tabular}{|l|}
\multicolumn{1}{|c|}{ Principais cuidados de Enfermagem aos pacientes portadores de LES } \\
\hline - Promover ambiente calmo para sono e repouso \\
\hline - Controlar rigorosamente o balanço hídrico \\
\hline - Verificar peso diariamente em jejum \\
\hline - Avaliar estado geral e nutricional \\
\hline - Manter níveis pressóricos adequados \\
\hline - Avaliar acessos venosos e outros dispositivos invasivos quanto a sinais flogísticos \\
\hline - Avaliar a dor e promover ambiente tranquilo \\
\hline - Evitar leitos com incidência direta de luz solar \\
\hline - Avaliar resultados de exames laboratoriais \\
\hline - Oferecer cremes a base de uréia para hidratação da pele \\
\hline - Incentivar e orientar sobre o autocuidado \\
\hline - Fornecer informações suficientes sobre a doença e o tratamento \\
\hline - Oferecer apoio emocional para enfrentamento das mudanças corporais \\
\hline
\end{tabular}


Em síntese, os cuidados de enfermagem aos pacientes portadores de LES internados na enfermaria de clínica médica eram complexos e necessitavam de avaliação contínua afim de resolver ou minimizar os problemas reais e potenciais identificados na clientela. A necessidade de implementar cuidados de enfermagem que visem a melhor qualidade de vida desse paciente é imprescindível para o sucesso na adesão ao tratamento, ainda que este seja intra-hospitalar. Dentre os inúmeros cuidados de enfermagem que podem ser ofertados, os mencionados acima foram os mais realizados na prática assistencial vivenciada pela enfermeira residente.

A promoção do ambiente calmo e tranquilo era feita diminuindo sons de televisão e luzes, o controle rigoroso do balanço hídrico era feito por meio de um instrumento próprio que contemplava o registro de todos os líquidos administrados e eliminados pelo paciente, a verificação do peso em jujum era feita através de uma balança disposta na enfermaria onde os pacientes eram pesados diariamente em jejum, a avaliação do estado geral e nutricional era feita por meio de visita diária a cada paciente com atenção para as queixas, ganho ou perda de peso e aceitação da dieta oferecida, a manutenção dos níveis pressóricos adequados era realizada através da aferição da pressão arterial regularmente a cada 6 horas, bem como a comunicação com a equipe médica em casos de hipotensão ou hipertensão, a avaliação dos acessos venosos e outros dispositivos invasivos era feita diariamente com atenção para correta fixação e data dos acessos venosos, bem como permeabilidade e sinais flogísticos (dor, edema, rubor, hiperemia, perda da capacidade funcional), a avaliação da dor era feita por meio da escala visual analógica e os relatos verbais de dor do paciente.

Dessa maneira, a experiência prática de atuação na assistência à saúde aos pacientes portadores de LES e os achados na literatura proporcionaram reflexões e discussões interessantes acerca do tema. Os pacientes acompanhados durante a prática assistencial eram do sexo feminino, de raça branca e com idade entre 20 e 40 anos, o que corrobora com a literatura que evidencia maior prevalência da doença em mulheres com idade entre 20 a 45 anos, porém confronta os dados onde a descendência afroamericana é a mais atingida. ${ }^{6}$

Os cuidados de enfermagem realizados na enfermaria de clínica médica também corroboram com os achados na literatura onde a preocupação com os problemas reais e potenciais do cliente eram voltados, sobretudo, ao controle de líquidos, controle de peso, controle da pressão arterial, avaliação do estado nutricional, avaliação da dor, avaliação dos dispositivos invasivos, entre outros. ${ }^{9}$

Um cuidado de enfermagem que não foi descrito na literatura, mas que foi realizado aos portadores de LES diz respeito a não acomodar o paciente em leitos com incidência direta de luz solar a fim de evitar o contato da pele do paciente com os raios ultravioletas para que não houvesse maior exacerbação da doença e dos sintomas cutâneos.

$\mathrm{O}$ cuidado de enfermagem citado nos artigos selecionados e que fala sobre o incentivo à prática de atividade física não era ofertado na prática assistencial aos portadores de LES da enfermaria, possivelmente pelo estado físico e emocional desses pacientes e não se adequava ao momento de internação. ${ }^{9,10}$

Além disso, outro dado relevante diz respeito à orientação do autocuidado ao paciente portador de LES, pois foi de grande importância para a adaptação do paciente em relação à situação de saúde vivenciada naquele momento e favorecia a adesão ao tratamento e ao enfrentamento dos efeitos colaterais dos medicamentos utilizados.

Como residente de enfermagem em clínica médica, pode-se perceber que para a melhor qualidade da assistência e dos cuidados ofertados, faz-se necessário um dimensionamento de pessoal adequado para as demandas dos pacientes e os recursos necessários para o estabelecimento do cuidado seguro e digno. Portanto é importante destacar que a instabilidade política no Estado do Rio de Janeiro e os sucessivos cortes no orçamento do hospital, contribuíram negativamente na aquisição de recursos humanos, não só da equipe de enfermagem, mas no corpo de trabalhadores de forma geral, além da diminuição dos recursos materiais imprescindíveis para uma assistência de qualidade.

É necessário também pontuar que a escassez de produção científica na área da enfermagem voltada aos cuidados de enfermagem para o paciente portador de LES reflete o pouco interesse da categoria em pesquisas voltadas para o Lúpus e seu manejo clínico pela equipe de enfermagem.

\section{Conclusão}

O cenário dos cuidados de enfermagem aos pacientes portadores de Lúpus Eritematoso Sistêmico é desafiador à medida que os pacientes internados possuem vários órgãos acometidos e isso torna a avaliação mais complexa e que também que a literatura pertinente aos cuidados de enfermagem ao paciente portador de LES é bastante escassa, o que torna este estudo relevante para os profissionais da enfermagem e sociedade.

É imprescindível destacar a Sistematização da Assistência de Enfermagem como ferramenta de intervenção, no sentido de promover a saúde e de prevenir danos, como é o caso dos pacientes crônicos. Ressaltase que o cuidado de enfermagem ao paciente lúpico está relacionado diretamente à redução de danos, ou seja, a redução do risco de complicações, possibilitando uma melhor qualidade de vida aos portadores de LES e, nesse sentindo, a Sistematização da Assistência de 
Enfermagem viabiliza os passos do enfermeiro com vistas ao atendimento seguro e digno para o paciente.

A experiência na enfermaria de clínica médica revelou que o paciente portador de LES tem grande potencial de agravamento se não for bem assistido por uma equipe multiprofissional capacitada, sobretudo a equipe de enfermagem, onde o enfermeiro tem o papel fundamental de prevenir complicações, minimizar riscos e atuar frente às vulnerabilidades do paciente respeitando sua individualidade e crenças pessoais.

No que se refere à limitação do estudo houve um baixo número de artigos publicados sobre os cuidados de enfermagem aos pacientes portadores de LES nos últimos cinco anos, somado à dificuldade de captação de dados para a descrição do relato de experiência, visto que não é uma modalidade de publicação de grande adesão das revistas científicas. Sendo assim, recomendamse pesquisas futuras para aprimorar as lacunas do conhecimento da enfermagem e o aprimoramento da assistência à saúde para essa clientela.

\section{Referências}

1.Vargas KS, Marco AR. Lúpus eritematoso sistêmico: aspectos epidemiológicos e diagnóstico. Rev Salus-Guarapuava 2009;3(1):15-22.

2. Secretaria de Atenção à Saúde (Brasil). Portaria nº ${ }^{\circ}$ 100, de 7 de fevereiro de 2013. Institui oprotocolo clínico e diretrizes terapêuticas do Lúpus Eritematoso Sistêmico. Diário Oficial da União 7 fev 2013; Seção 1.

3. Borba EF, Latorre LC, Brenol JCT, Kayser C, Silva NA, Zimmermann $\mathrm{AF}$, et al. Consenso de lúpus eritematoso sistêmico. RevBras de Reumatol2008;48:196-207.

4.Skare TL, Dagostini JS, Zanardi PI, Nisihara RM. Infecções e lúpus eritematoso sistêmico. Rev. Einstein2016;1(14):47-51.

5.Sociedade Brasileira de Reumatologia [homepage na internet]. Lúpus Eritematoso Sistêmico (LES)[acesso em 05 jul 2018]. Disponível em: https://www.reumatologia.org.br/doencas-reumaticas/lupus-eritematososistemico-les/

6.Bireme. Biblioteca Virtual em Saúde. Programa Nacional Telessaúde. Núcleo de Telessaúde Rio Grande do Sul. O que é Lúpus Eritematoso? 2014[acesso em 2 set 2017]. Disponível em: http://aps.bvs.br/aps/o-que-elupus-eritematoso

7. Horta WA. Processo de Enfermagem. Rio de Janeiro: Guanabara Koogan; 2015.

8. Conselho Nacional de Saúde (Brasil). Resolução n. 466, de 12 de dezembro de 2012. Diretrizes e normas regulamentadoras de pesquisas envolvendo seres humanos. Diário Oficial da União 12 dez 2012; Seção 1.

9. Almeida RA, Pesqueno GA, Almeida FCA, Bastos RAA, Barros MES. Aplicando o Processo de Enfermagem no Cuidar de um Paciente com Lúpus Eritematoso Sistêmico.R bras ci Saúde 2013; 17(2):121-126.

10. Robinson M, Cook SS, Currie LM. Systemic lupus erythematosus: A genetic review for advanced practice nurses. American Academy of Nurse Practitioners2011;23:629-637.

11. Hospital Universitário Pedro Ernesto [homepage da internet]. Histórico e missão. [acesso em 20 jul 2019] . Disponível em: http://www.hupe.uerj.br/ hupe/Institucional/IN_historico.php 